\title{
GRB HOST GALAXIES: A FASCINATING RESEARCH FIELD
}

\author{
S. Klose ${ }^{1}$
}

\begin{abstract}
Because of time constraints, there was no discussion on GRB host galaxies. Nevertheless, it is worth to extract the main points from the talks and posters presented during the conference.
\end{abstract}

\section{Substantial activity in the field}

The overwhelming majority of observational data concerns the hosts of long bursts (e.g., Savaglio et al. 2009; see also the www page of the GHostS project ${ }^{2}$ ), and meanwhile even redshift trends of host galaxy properties can be discussed (Savaglio, these proceedings). There is a lively discussion ongoing in the community whether or not long GRB hosts trace and quantify the evolution of the cosmic star-formation rate (SFR) and what the relations between mass, metallicity, and SFR tell us here (Graham et al.; Niino et al.; Savaglio et al., these proceedings; Mannucci et al. 2011). More and more spectroscopic data of GRB hosts are published, either based on afterglow follow-up observations (e.g., Christensen et al. 2011) or based on host galaxy studies. At least in the latter case it is obvious that this mainly remains a job for 8-m class optical telescopes. Observations with VLT/X-Shooter are particularly fascinating here (Vergani et al., these proceedings; Vergani et al. 2011).

Increasing interest develops towards a study of those hosts that harbor dark bursts, and the MIR/FIR/submm band is particularily intriguing here (Palazzi et al., these proceedings). This holds in particular after it has been discovered that a substantial population of dark bursts might be hosted by extremely red, globally dust-enshrouded objects (Rossi et al. 2012), and that the hosts of the dustiest GRBs point to a population of luminous, massive, and chemically evolved galaxies (Krühler et al. 2011), which are very different from the majority of GRB hosts. Obviously, there is a lot to investigate here.

On the far site, the hosts of high- $z$ events remain fascinating (Basa et al. 2012), in particular since basically all of them remain undetected even for HST

\footnotetext{
1 Thüringer Landessternwarte Tautenburg, 07778 Tautenburg, Germany

${ }^{2}$ http://www.grbhosts.org/
} 
(Tanvir et al., these proceedings; Tanvir et al. 2012). It seems we have to await here the next generation of ground and space-based optical telescopes in order to learn more about these galaxies (or these galaxy building blocks). Given that GRBs are the potential messengers of the first stars in the universe, chances are not small that these hosts will be among the first targets for these future telescopes.

On the near site, we might divide between the hosts of near-by GRB-SNe and the hosts of short bursts. The host of SN 1998bw still represents the nearest GRB host galaxy; it promises the best achievable data quality with respect to angular resolution. Substantial work has already been done here (e.g., Christensen et al. 2008; Fynbo et al. 2000; Michałowski et al. 2009), and also in the case of other $z<0.1$ GRBs the observational data can be of remarkable scientific quality (e.g., Thöne et al. 2008). On the contrary, after some early enthusiasm in the years 2005/06 (e.g., Fox et al. 2005; Gehrels et al. 2005), the following years have not given us so many nearby short burst host galaxies (the most remarkable exception is GRB 080905A at $z=0.122$; Rowlinson et al. 2010). Most of these hosts are at cosmological distance (e.g., Berger 2009). Hopefully, more high-resolution data will be obtained with HST soon.

\section{Looking forward}

The GRB field is rapidly developing and host galaxies are becoming an important observational target.

A better understanding of the nature of the long and short burst progenitors requires a deeper look into their birth places, deep in angular resolution in particular. For long bursts this mainly concerns the metallicity issue and the decision between the single and the binary star scenario. For this task, the host of SN 1998bw remains the best target in our days. More such near-by events are needed, but given the GRB redshift distribution, we might have to wait for another ten years, or so, to get this picture right. For short bursts, distance is the main challenge, too. Some open questions are: What are the characteristic ages of the short burst progenitors, are they representing a young or an old stellar population? Well possible that just a single nearby event in the coming years will bring us a big step forward in our understanding of the progenitor properties.

Following the very successful TOUGH survey with the ESO/VLT (Jakobsson et al., these proceedings) also large amounts of Keck data become public now (Perley et al., these proceedings), while HST remains the work horse for unprecedented deep and high-resolution host galaxy data (Fruchter et al., these proceedings). ALMA and the Herschel satellite, following Spitzer, have opened the window into the MIR/FIR/submm regime (Sokolov et al., these proceedings). Together with VLA, ATCA, WSRT, and GMRT this offers the possibility of a detailed characterization of the SED of host galaxies from radio to UV wavelengths, with the goal to derive the SFR and other galaxy parameters (e.g., Hunt et al. 2011; Michałowski et al. 2012; Svensson et al. 2012). We can expect a fascinating progress on GRB host galaxy studies in the coming years. 


\section{References}

Basa, S., Cuby, J.G., Savaglio, S., et al., 2012, A\&A, 542, 103

Berger, E., 2009, ApJ, 690, 231

Christensen, L., Vreeswijk, P.M., Sollerman, J., et al., 2008, A\&A, 490, 45

Christensen, L., Fynbo, J.P.U., Prochaska, J.X., et al., 2011, ApJ, 727, 73

Fox, D., Frail, D.A., Price, P.A., et al., 2005, Nature, 437, 845

Fynbo, J.P.U., Holland, S., Andersen, M.I., et al., 2000, ApJ, 542, 89

Gehrels, N., Sarazin, C.L., O'Brien, P.T., et al., 2005, Nature, 437, 851

Hunt, L., Palazzi, E., Rossi, A., et al., 2011, ApJ, 736, 36

Krühler, T., Greiner, J., Schady, P., et al., 2011, A\&A, 534, 108

Mannucci, F., Salvaterra, R., \& Campisi, A., 2011, MNRAS, 414, 1263

Michałowski, M.J., Hjorth, J., Malesani, D., et al., 2009, ApJ, 693, 347

Michałowski, M.J., Kamble, A., Hjorth, J., et al., 2012, ApJ, 755, 85

Rossi, A., Klose, S., Ferrero, P., et al., 2012, A\&A, 545, 77

Rowlinson, A., Wiersema, K., Levan, A.J., et al., 2010, MNRAS, 408, 383

Savaglio, S., Glazebrook, K., \& Le Borgne, D., 2009, ApJ, 691, 182

Svensson, K.M., Levan, A.J., Tanvir N.R., et al., 2012, MNRAS, 421, 25

Tanvir, N.R., Levan, A.J., Fruchter, A.S., et al., 2012, ApJ, 754, 46

Thöne, C.C., Fynbo, J.P.U., Östlin, G., et al., 2008, ApJ, 676, 1151

Vergani, S.D., Flores, H., Covino, S., et al., 2011, A\&A, 535, 127 
